\title{
Combating Diabetes and its Emerging Complications Utilizing Natural Phytochemicals
}

\author{
Karim Raafat*
}

\section{Karim Raafat* \\ Department of Pharmaceutical Sciences, Faculty of Pharmacy, Beirut Arab University, Beirut 115020, LEBANON. \\ Correspondence \\ Dr. Karim Raafat}

Department of Pharmaceutical Sciences, Faculty of Pharmacy, Beirut Arab University (BAU), Beirut, LEBANON.

Phone no : +961 1300110

E-mail: k.raafat@bau.edu.lb

\section{History}

- Submission Date: 15-10-2018;

- Review completed: 12-12-2018;

- Accepted Date: 18-12-2018.

DOI : 10.5530/pj.2019.11.69

Article Available online http://www.phcogj.com/v11/i3

\section{Copyright}

(c) 2019 Pharmacognosy Journal. This is an open-access article distributed under the terms of the Creative Commons Attribution 4.0 International license.

\begin{abstract}
Introduction: Natural phytochemicals are considered a primary health care measure for many chronic diseases and for assurance of urban health and wellbeing. Diabetes and its related complications are major chronic diseases increasingly threatening human health and wellbeing. Till date, these diseases are not fully managed by the current therapies. Thus, there is an increasing need to find more safe and efficient therapies for diabetes and its related complications. Methods: An evidence-based review of the novel phytotherapies to diabetes and its related complications and discussing their main mechanisms of actions are the main aims of the current study. Data collection were done for the phytotherapies that were extracted, chromatographically standardized, fractionated and the main effective compounds were isolated and evaluated for their potentials against diabetes and its related complications. Special emphasis was given for the extracts and their isolated phytochemicals that had significant hypoglycemic and antinociceptive effects towards diabetes and its related complications, particularly diabetic-neuropathy. Results: The phytochemicals main mechanisms of action were found to be mainly due to their insulin secretagogue, beta-cells regeneration, anti-oxidant potentials. Conclusion: Therefore, clinicians should consider natural phytochemicals when treating chronic diseases and when reassuring better urban health and wellbeing.

Key words: Combating Diabetes, Emerging Complication, Natural Phytochemicals Mechanism of action, Complementary medicine.
\end{abstract}

\section{INTRODUCTION}

Natural phytochemicals are considered a primary health care measure for many chronic diseases and for assurance of urban health and wellbeing. ${ }^{1}$ Diabetes (DM) and its related complications are major chronic diseases increasingly threatening human health and wellbeing. ${ }^{2}$

Type $1 \mathrm{DM}$ is ameliorated with insulin, exercise and dietary changes. On the other hand, type $2 \mathrm{DM}$ might be ameliorated with oral hypoglycemics, reduction of weight or changes in diet. ${ }^{3}$ Till date, these diseases are not fully managed by the current therapies. ${ }^{3}$ Thus, there is an increasing need to find more safe and efficient therapies for diabetes and its related complications.

Diabetes mellitus is associated with vascular-disorders. Current reports have accompanied hyperglycemia and oxidative stress and its relation to diabetic complications. Natural phytochemicals are famous for their free radical scavenging potentials which might be involved in amelioration of diabetes and its complication. ${ }^{4}$

Therefore, an evidence-based review of the novel phytotherapies to diabetes and its related complications and discussing their main mechanisms of actions are the main aims of the current study.

\section{MATERIALS AND METHODS}

\section{Search strategy}

The investigation in the primary-literature was done between August and September 2018. A web-based search was done in SCOPUS and PUBMED. Databases were searched using a search-strategy done by combining the following keywords: diabetes or diabetic neuropathy or diabetes diagnosis or diabetes current-therapies or diabetes mechanisms or diabetes extract or diabetes phytotherapies or diabetes therapy-recommendations. The related research article references were searched for the relevant tertiary and secondary literature.

\section{Inclusion and Exclusion criteria}

The subsequent inclusion and exclusion criteria were used; paper type: 1ry, 2ry or 3ry literature, printed in English, in well-reputed published books or peer-reviewed journals. The study design included pre-clinical or clinical models. The selection of natural phytochemicals was established on their potentials in amelioration of diabetes in clinical or pre-clinical models.
Cite this article: Raafat K. Combating Diabetes and its Emerging Complications Utilizing Natural Phytochemicals. Pharmacog J. 2019;11(3):445-9. 


\section{Quality-assessment}

The included researches quality was evaluated by a standardized-tool. This tool consisted of 8 factors: (A) The sample was representative to the population, (B) the design of studies, (C) external-factors control (D) blinding (E) data-collection consistency, $(\mathrm{F})$ the criteria for exclusion and inclusion, $(\mathrm{G})$ reliability of the method and $(\mathrm{H})$ analyses of the method. The researches were included in this study in the condition of all criteria were fulfilled. ${ }^{5}$

\section{Data-synthesis}

With the purpose of combing the retrieved results, a narrative synthesis technique was used. The 1ry research characteristics were evaluated using a pre-set procedure and the subsequent data has been extracted: natural phytochemicals' family and name, the part used, scope of the study, most bioactive ingredients, uppermost dose used, analyses-methods, administration method, clinical model or pre-clinical, existence of positive-control and its administration route, the results-section, diabetes limitations and indications.

\section{RESULTS AND DISCUSSION}

\section{Current-therapies}

As from a pharmacological-perspective, conventional-drugs ameliorating diabetes are summarized in Table $1 .^{6,7}$ Despite the lack of full information from meta-analyses, various conventional-drugs showed clinically-sounding effects on diabetes and its related symptoms and complications (Table 1).

\section{Natural Phytochemicals}

Currently, natural phytochemicals were used as a complementary therapy for the amelioration of DM. ${ }^{8-10}$ Many researchers presented proof underlying the beneficial potentials of natural phytochemicals towards DM (Table 2).

The $R$. ribes showed to be among the most effective natural phytochemicals. Bio-guided separation techniques have shown evidence that rutin significantly decreased hyperglycemia (Table 2) and this effect suggested to be due to the increase of natural-antioxidant effect, serum catalase. ${ }^{11}$ However, more full clinical interventions are needed to strengthen its

Table 1: DM current therapies.

\begin{tabular}{|c|c|c|}
\hline Group & Individuals & Mechanism \\
\hline \multirow{4}{*}{ Sulfonylurea } & Glibenclamide & \multirow{4}{*}{ Insulin secretagogue (Type $2 \mathrm{DM}$ ) } \\
\hline & Gliclazide & \\
\hline & Glipizide & \\
\hline & Gliemepiride & \\
\hline \multirow{2}{*}{$\begin{array}{l}\text { Meglitinide } \\
\text { analogues }\end{array}$} & Repaglinide & \multirow{2}{*}{ Insulin secretagogue (Type 2 DM) } \\
\hline & Nateglinide & \\
\hline & & $\downarrow$ hepatic glucose production \\
\hline Biguanides & Metformin & $\begin{array}{c}\downarrow \text { intestinal-absorption of glucose and } \\
\uparrow \text { insulin sensitivity } \\
\text { (Type } 1 \text { and } 2 \text { DM) }\end{array}$ \\
\hline \multirow{2}{*}{ Thiazolidinediones } & Rosigliltazone & \multirow{2}{*}{$\begin{array}{c}\uparrow \text { Adipogenesis and fatty-acid uptake } \\
\text { (Type } 2 \mathrm{DM} \text { ) }\end{array}$} \\
\hline & Pioglitazone & \\
\hline $\begin{array}{l}\text { Alpha-glycosidase } \\
\text { inhibitors }\end{array}$ & Acarbose & $\begin{array}{l}\text { Alpha-glycosidase inhibitors } \\
\text { (Type } 1 \text { and } 2 \text { DM) }\end{array}$ \\
\hline Insulin & Insulin & $\begin{array}{l}\text { Control the metabolism of } \\
\text { carbohydrates, fats and protein } \\
\text { (Type } 1 \mathrm{DM})\end{array}$ \\
\hline
\end{tabular}

\begin{tabular}{|c|c|c|c|c|c|}
\hline 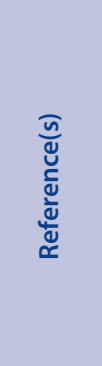 & 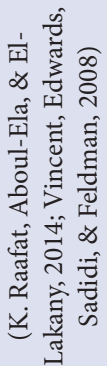 & 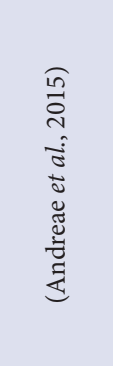 & 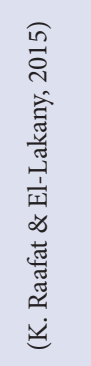 & 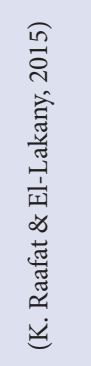 & 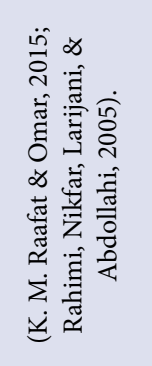 \\
\hline 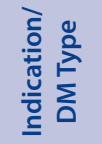 & 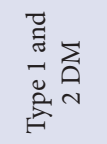 & \begin{tabular}{l}
$\sum_{n}$ \\
$\sim$ \\
$\tilde{z}$ \\
\multirow{2}{*}{}
\end{tabular} & 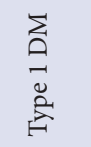 & $\begin{array}{l}\sum_{\Delta} \\
\vec{\Xi} \\
\stackrel{\Xi}{=}\end{array}$ & 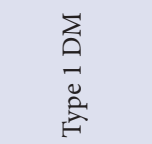 \\
\hline 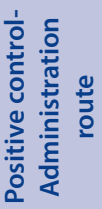 & 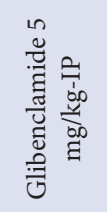 & | & 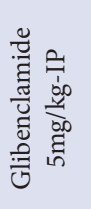 & 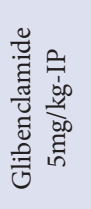 & 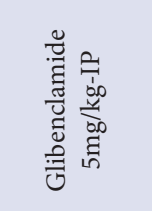 \\
\hline 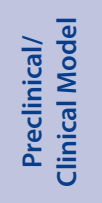 & 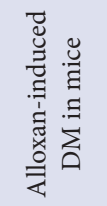 & 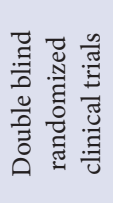 & 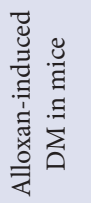 & 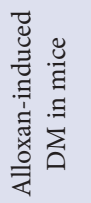 & 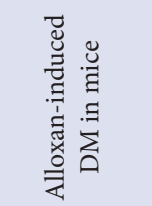 \\
\hline 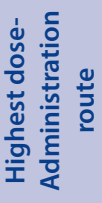 & 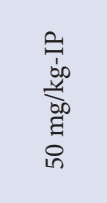 & 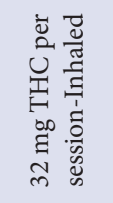 & 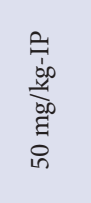 & 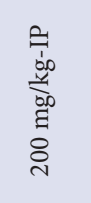 & 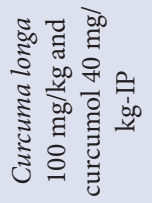 \\
\hline 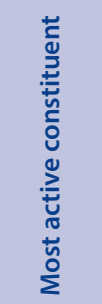 & 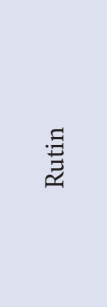 & 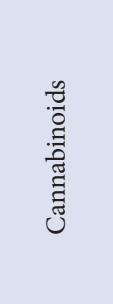 & 营 & 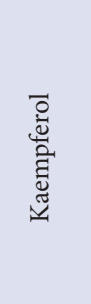 & 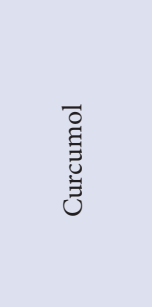 \\
\hline 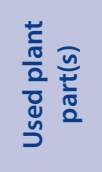 & $\begin{array}{l}\stackrel{0}{0} \\
0 \\
\approx\end{array}$ & $\begin{array}{l}\text { 岕 } \\
\text { 苛 }\end{array}$ & $\begin{array}{l}\stackrel{5}{0} \\
0 \\
\approx\end{array}$ & 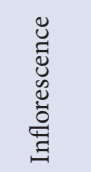 & $\begin{array}{l}0 \\
\stackrel{0}{0} \\
\approx\end{array}$ \\
\hline 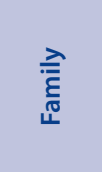 & 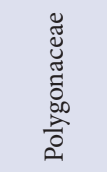 & 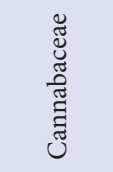 & 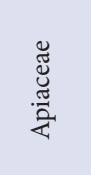 & 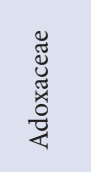 & 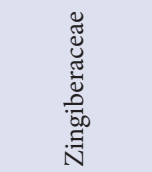 \\
\hline 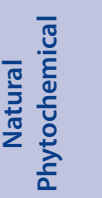 & 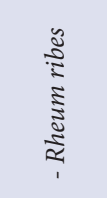 & 胥 & 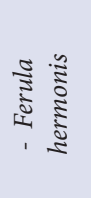 & 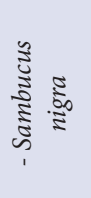 & 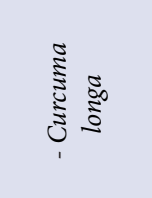 \\
\hline
\end{tabular}




\begin{tabular}{|c|c|c|c|c|c|c|c|c|c|c|c|}
\hline 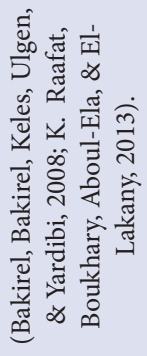 & 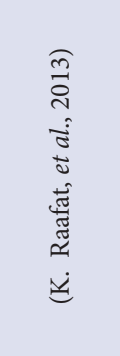 & 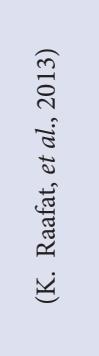 & 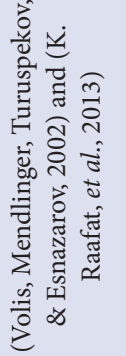 & 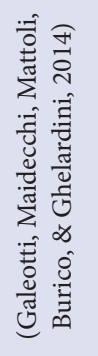 & 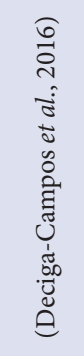 & 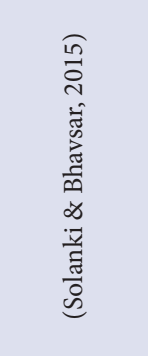 & 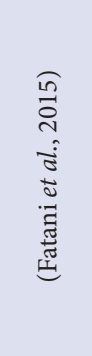 & 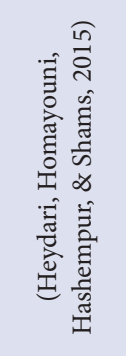 & 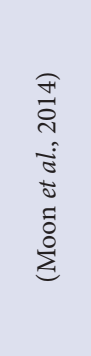 & 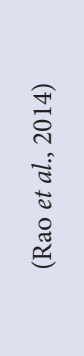 & 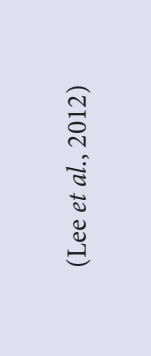 \\
\hline 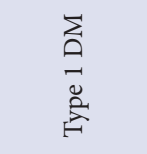 & 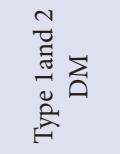 & 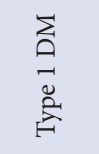 & 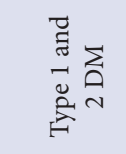 & 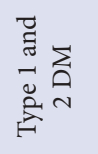 & 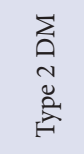 & 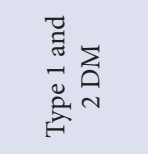 & 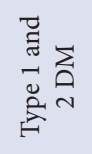 & 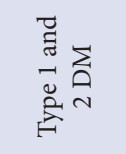 & 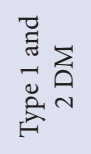 & 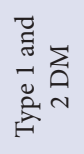 & 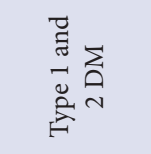 \\
\hline 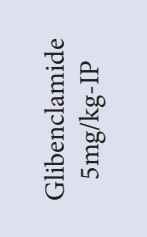 & 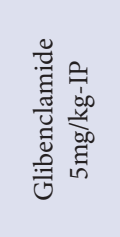 & 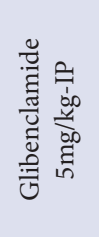 & 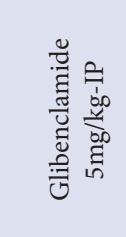 & I & $\begin{array}{l}\stackrel{a}{1} \\
\stackrel{g}{\Xi} \\
\vdots \\
\Xi\end{array}$ & 1 & 1 & 1 & 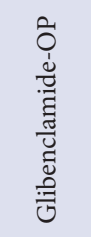 & I & 1 \\
\hline 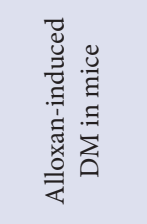 & 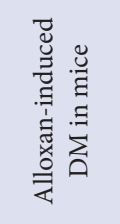 & 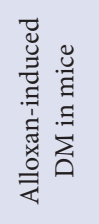 & 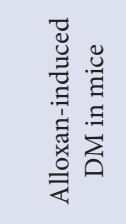 & 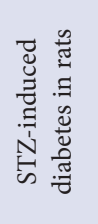 & 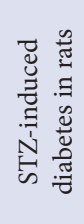 & 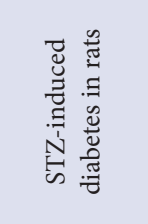 & 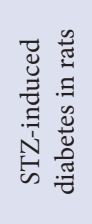 & 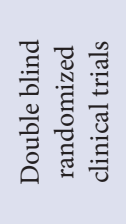 & 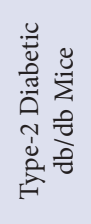 & 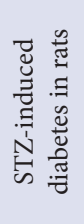 & 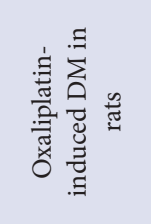 \\
\hline 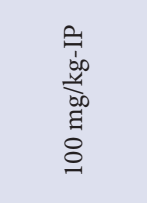 & 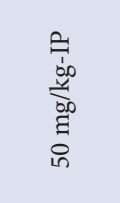 & 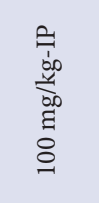 & 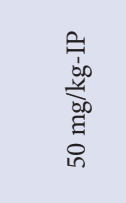 & 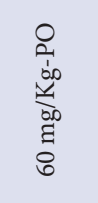 & 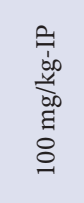 & 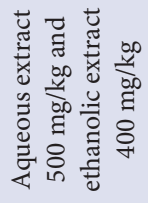 & 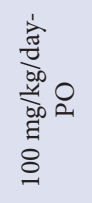 & 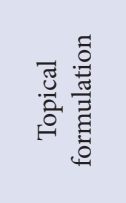 & 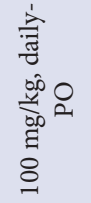 & 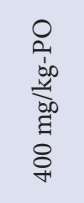 & 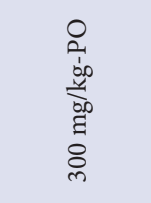 \\
\hline 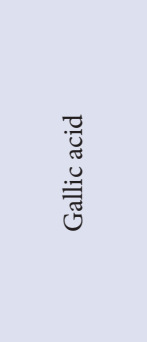 & 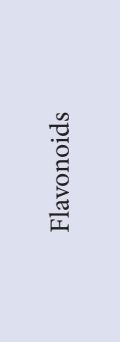 & 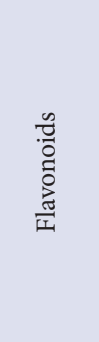 & 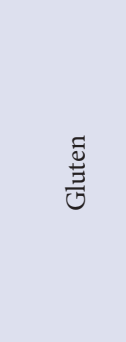 & 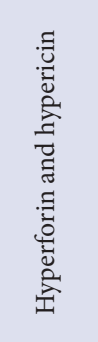 & 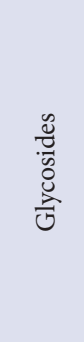 & 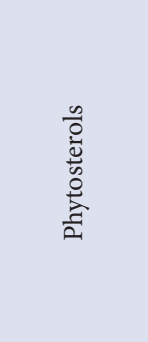 & 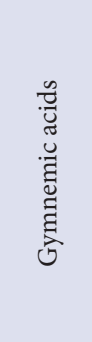 & 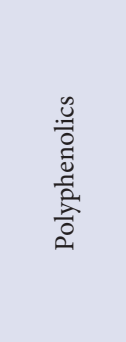 & 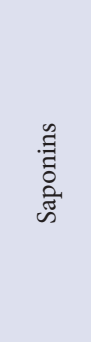 & 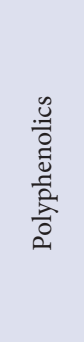 & 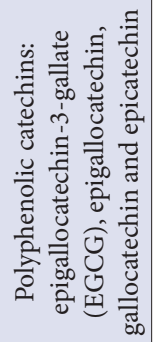 \\
\hline 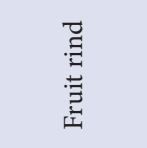 & 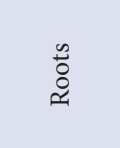 & 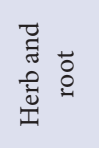 & 䨠 & 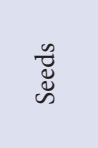 & $\begin{array}{l}0 \\
\stackrel{0}{0} \\
\approx\end{array}$ & $\begin{array}{l}\text { चू } \\
\text { है } \\
\text { ज्ञ } \\
\text { के }\end{array}$ & $\stackrel{\mathscr{J}}{\leftrightarrows}$ & 蚟 & 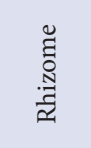 & 离 & 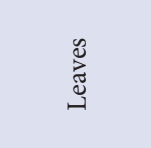 \\
\hline 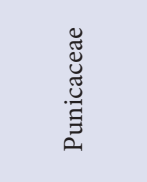 & 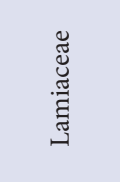 & 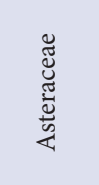 & 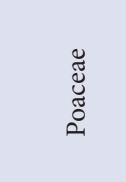 & 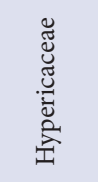 & 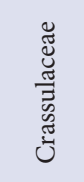 & 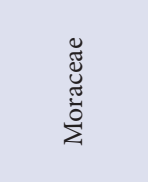 & 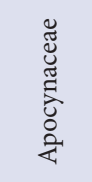 & 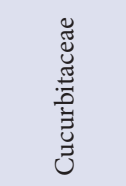 & 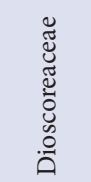 & 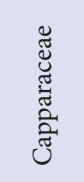 & $\begin{array}{l}\stackrel{\Xi}{\Xi} \\
\widetilde{\Xi} \\
\text { F }\end{array}$ \\
\hline 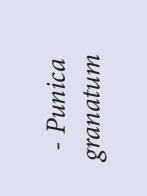 & 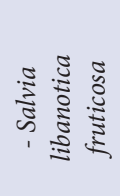 & 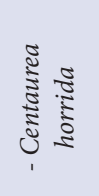 & 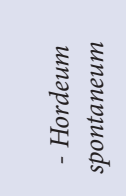 & 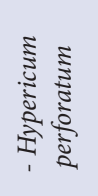 & 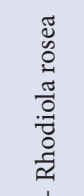 & 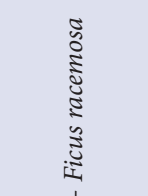 & 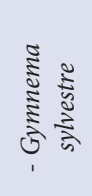 & 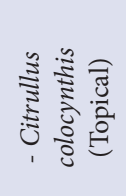 & 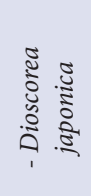 & 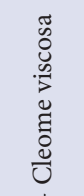 & 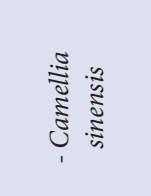 \\
\hline
\end{tabular}


utilization. The C. sativa showed potent hypoglycemic effects (Table 2). Randomized double-blind clinical trials showed that THC administered by inhalation significantly decreased Blood Glucose Level (BGL) and ameliorated diabetic neuropathy by inhibition of peripheral and central sensitization. ${ }^{12,13}$

Moreover, F. hermonis has shown potent hypoglycemic activity (Table 2). Ferutinin has shown to be $F$. hermonis most active constituent responsible for the amelioration of DM. The potential antioxidant effect of ferutinin might be the underlying cause of management of DM. ${ }^{14}$ The S. nigra showed potent antihyperglycemic activity (Table 2). Even though there is no detailed clinical-trial till date was reported, Kaempferol has shown to be its most bioactive substance and Kaempferol anti-oxidative stress potentials might be responsible for this effect. ${ }^{14}$ The study performed on C. longa indicated its potentials against DM and curcumol might be its key constituent responsible for the alleviation of DM4 (Table 2). More deep clinical-trials are needed to completely-understand C. longa hypoglycemic mechanism.

The investigation on P. granatum (Table 2) showed its promising hypoglycemic activities on Type 1 and 2 DM. The antioxidative stress activity of gallic acid might be responsible for this activity. ${ }^{15}$ Other studies including S. fruticosa, C. horrida and $H$. spontaneum has shown hypoglycemic activity, but also lacks the clinical evidence.16 H. perforatum, $R$. rosea, $F$. racemosaand $G$. sylvestre (Table 2 ) have showed significant antihyperglycemic potentials. These findings reflect positive outcomes for further investigation. ${ }^{17-19}$ Inside the inclusion-criterion of the current study, C. colocynthis, D. japonica, C. viscosa and green tea have been appropriately researched for the amelioration of DM. More studies are needed to ascertain their potentials on the overall quality of life..$^{20-23}$

Generally, natural phyto-chemicals showed to be effective and welltolerated by their in vivo-models of DM. Serious side-effects of these phyto-chemicals did not show as an arising problem. Nevertheless, natural phyto-chemicals require additional clinical-trials to show possible sideeffects and to support natural phyto-chemicals clinical use.

\section{CONCLUSION}

The phytochemicals main mechanisms of action were found to be mainly due to their insulin secretagogue, beta-cells regeneration, anti-oxidant potentials. Therefore, clinicians should consider natural phytochemicals when treating chronic diseases and when reassuring better urban health and wellbeing.

\section{ACKNOWLEDGEMENT}

Thanks to Mrs. G. Onsy for English proof-reading the current manuscript.

\section{CONFLICT OF INTEREST}

The author declares no conflicts of interest.

\section{ABBREVIATIONS}

BGL: Blood glucose level; DM: Diabetes mellitus; DN: Diabetic neuropathy; HbA1c: Glycated hemoglobin; R. ribes: Rheum ribes; T1DM: Type 1 diabetes mellitus; T2DM: Type 2 diabetes mellitus.

\section{REFERENCES}

1. Raafat K, et al. Neuropathic Pain: Literature Review and Recommendations of Potential Phytotherapies. Pharmacognosy Journal. 2017;9(4).

2. Saleh FA, et al. Phytochemical Analysis of Nigella sativa L. Utilizing GC-MS Exploring its Antimicrobial Effects against Multidrug-Resistant Bacteria. Pharmacognosy Journal. 2018;10(1).

3. Ziegler D. Can diabetic polyneuropathy be successfully treated?. MMW Fortschr Med. 2010;152(9):64-8.

4. Raafat KM, Omar AG. Phytotherapeutic activity of curcumol: Isolation, GC-MS identification and assessing potentials against acute and subchronic hyperglycemia, tactile allodynia and hyperalgesia. Pharm Biol. 2015;1-11.

5. MacRae $\mathrm{S}$, et al. Diabetes in people with intellectual disabilities: A systematic review of the literature. Res Dev Disabil. 2015;47:352-74.

6. Carter GT, Galer BS. Advances in the management of neuropathic pain. Phys Med Rehabil Clin N Am. 2001;12(2):447-59.

7. WHO. Global guideline for type 2 diabetes. Diabetes Res Clin Pract. 2014;104(1):1-52.

8. $\mathrm{Xu} \mathrm{HB}$, et al. Chinese herbal medicine in treatment of diabetic peripheral neuropathy: a systematic review and meta-analysis. J Ethnopharmacol. 2012;143(2):701-8

9. Wu QL, Liang XC. Survey of current experimental studies of effects of traditional Chinese compound recipe on diabetic peripheral neuropathy. Zhongguo Zhong Yao Za Zhi. 2007;32(9):775-8.

10. Raafat K, El-Lakany A. Phytochemical and Antinociceptive Investigations of Anemone coronaria Active Part Ameliorating Diabetic Neuropathy Pain. PMIO. 2018;5(01):e5-e13.

11. Raafat $\mathrm{K}$, et al. Alloxan-induced diabetic thermal hyperalgesia, prophylaxis and phytotherapeutic effects of Rheum ribes L. in mouse model. Arch Pharm Res. 2014.

12. Andreae MH, et al. Inhaled Cannabis for Chronic Neuropathic Pain: A Metaanalysis of Individual Patient Data. J Pain. 2015;16(12):1221-32.

13. Lynch ME, et al. A double-blind, placebo-controlled, crossover pilot trial with extension using an oral mucosal cannabinoid extract for treatment of chemotherapy-induced neuropathic pain. J Pain Symptom Manage. 2014;47(1):166-73.

14. Raafat K, El-Lakany A. Acute and subchronic in-vivo effects of Ferula hermonis $\mathrm{L}$. and Sambucus nigra $\mathrm{L}$. and their potential active isolates in a diabetic mouse model of neuropathic pain. BMC Complement Altern Med. 2015;15(1):257.

15. Raafat K, Samy W. Amelioration of Diabetes and Painful Diabetic Neuropathy by Punica granatum L. Extract and Its Spray Dried Biopolymeric Dispersions. Evid Based Complement Alternat Med. 2014;2014:180495.

16. Raafat $\mathrm{K}$, et al. Endogenous Lebanese Plants Treating Diabetes and Related Complications. Nat Prod Chem Res. 2013;1(3):112-20.

17. Deciga-Campos $M$, et al. Antihyperalgesic Activity of Rhodiola rosea in a Diabetic Rat Model. Drug Dev Res. 2016;77(1):29-36.

18. Solanki ND, Bhavsar SK. An evaluation of the protective role of Ficus racemosa Linn. in streptozotocin-induced diabetic neuropathy with neurodegeneration. Indian J Pharmacol. 2015;47(6):610-5.

19. Fatani AJ, et al. Neuroprotective effects of on streptozotocin-induced diabetic neuropathy in rats. Exp Ther Med. 2015;9(5):1670-8.

20. Heydari M, et al. Topical Citrullus colocynthis (bitter apple) extract oil in painful diabetic neuropathy: A double-blind randomized placebo-controlled clinical trial. J Diabetes. 2015.

21. Moon E, et al. Dioscorea Extract (DA-9801) Modulates Markers of Peripheral Neuropathy in Type 2 Diabetic db/db Mice. Biomol Ther (Seoul). 2014;22(5):445-52.

22. Rao BS, et al. Effects of Cleome viscosa on hyperalgesia, oxidative stress and lipid profile in STZ induced diabetic neuropathy in Wistar rats. Pak J Pharm Sci. 2014;27(5):1137-45.

23. Lee JS, et al. Effect of green tea extracts on oxaliplatin-induced peripheral neuropathy in rats. BMC Complement Altern Med. 2012;12(1):124. 
GRAPHICAL ABSTRACT

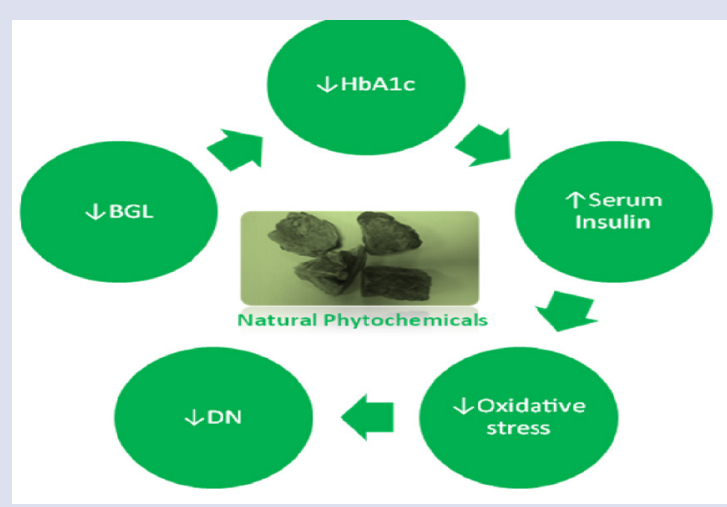

\section{SUMMARY}

- The phytochemicals main mechanisms of action were found to be mainly due to their insulin secretagogue, beta-cells regeneration, anti-oxidant potentials. Therefore, clinicians should consider natural phytochemicals when treating chronic diseases and when reassuring better urban health and wellbeing.

\section{ABOUT AUTHORS}

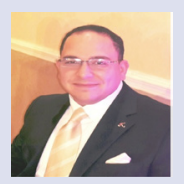

Dr. Karim M. Raafat is an Associate Professor of Phytochemistry and Pharmacognosy at Faculty of Pharmacy, Beirut Arab University. He has completed his PhD from German University in Cairo, New Cairo, Egypt, under the channel system and joint supervision scheme between The German University in Cairo (GUC) and Johann Wolfgang Goethe-University Frankfurt, Germany and postdoctoral studies from Beirut Arab University (BAU) and German University in Cairo. He is a visiting Scientist of Johann Wolfgang Goethe-University, Frankfurt, Germany. He is the Head of Phytochemistry Research Team and Junior Research Team, BAU, Lebanon. He has published more than seventy books, book chapters, patent and peer reviewed journal articles and presentations in scientific conferences. He has been honored with several national and international awards in the scientific field and public service. 